\section{Detection and Evaluation of Non-Recombinants in cDNA Libraries by Multi- ple Cloning Region PCR}

\author{
BioTechniques 32:88-92 (January 2002)
}

\section{ABSTRACT}

Bacteriophages that are routinely used in cDNA libraries do not require any biological selection for forming plaques. Thus parental non-recombinant phages are always found in variable proportions together with recombinant ones in all cDNA libraries. The presence of non-recombinants in significant proportions dilutes the abundance of rare cDNA species and makes library screening difficult. If the exact proportion of non-recombinants in a library were known, then one would screen proportionately more plaques to get a positive clone. In the absence of such information, screening is conventionally conducted on a number that is based on the titer of the library. We have devised a method using the flanking sequences from either side of the multiple cloning region (MCR) of all $\lambda$ phage vector derivatives as primers for PCR amplification. A non-recombinant phage produces a fragment equal to the size of the MCR, whereas a recombinant phage produces a fragment larger than the MCR, which is an MCR+ fragment. All cDNA libraries that we have studied show the presence of the MCR fragment (indicating nonrecombinants) at variable proportions ranging between $6 \%$ and $36 \%$ of the total phages present. We also show that their presence negatively influences the retrieval of target $c D N A$ sequences.

\section{INTRODUCTION}

Screening of cDNA libraries is very important for isolating transcripts of genes that regulate cellular functions and integrity. Several derivatives of $\lambda$ bacteriophage with improved cloning features have been in use as cloning vectors for many years $(2-4,8,9)$. In practice, however, parental non-recom binants produce a large number of plaques $(3,6)$, possibly because of frame-shift mutations (5). Phage titering remains the only method for evaluating the strength of a cDNA library, although, in reality, it only displays the number of plaque-forming bacteriophages rather than the actual number of recombinants in the library. The success of cloning a desired cDNA species depends directly on the complete representation of all mRNAs present at different abundance in the library. Non-recombinants, if present in significant numbers, lower the overall mRNA representation in the library and may underrepresent rare mRNA species. Thus, the total number of plaques to be screened as calculated by conventional methods for cloning of a low abundant cDNA might be less than are actually required. Consequently, screening of an inappropriate number of plaques may lead to confusion, costing more time and money because of repeated screenings. Therefore, there is a need for an accurate and convenient method that can identify and evaluate the exact proportion of non-recombinants in a cDNA library before it is used for cloning.

\section{MATERIALS AND METHODS}

\section{cDNA Library Construction}

cDNA was synthesized from the HeLa cell line and normal human placental mRNA using a linker-primer [oligo(dT)-XhoI] from the Zap-cDNA ${ }^{\circledR}$ synthesis kit (Stratagene, La Jolla, CA, USA). cDNA library construction and determination of titer was done follow ing the manufacturer's instructions. Routine blue/white plaque screenings were conducted to determine the $\mathrm{pfu} / \mu \mathrm{g}$ of vector arms.

\section{Phage-Recombinant and Non-Recombinant Control}

A 653-bp pBR322 fragment was cloned into the Zap ${ }^{\circledR}$ phage vector (Stratagene), confirmed by DNA sequencing, and used as a phage recombinant control in all multiple cloning region (MCR)-PCR amplifications. The parental Zap phage vector was used as a phage non-recombinant control.
Plasmid-Recombinant and NonRecombinant Control

A 560-bp cDNA fragment was cloned into pGEM ${ }^{\circledR}-7 Z f(-)$ plasmid (Promega, Madison, WI, USA), confirmed by DNA sequencing, and used as plasmid recombinant control. The parental pGEM-7Zf(-) plasmid was used as non-recombinant control.

\section{MCR-PCR}

Aliquots of the cDNA libraries were boiled for $5 \mathrm{~min}$, cooled on ice, microcentrifuged at $14000 \times g$ for $10 \mathrm{~min}$, and the supernatant used for MCR-PCR after DNA estimation. Plasmid DNA was used without pretreatment. The reaction mixture $(50 \mu \mathrm{L})$ consisted of $10 \mathrm{pmol}$ each primer, $50 \mu \mathrm{M}$ each dNTP, 1 U Taq DNA polymerase (Amersham Biosciences, Piscataway, NJ, USA) in PCR buffer (20 mM Tris-HCl, $\mathrm{pH} 8.4,50$ $\mathrm{mM} \mathrm{KCl}, 1.5 \mathrm{mM} \mathrm{MgCl}_{2}$ ). The PCR was carried out on a model TC-1 DNA thermal cycler from Applied Biosystems (Foster City, CA, USA) with an initial denaturation at $94^{\circ} \mathrm{C}$ for $4 \mathrm{~min}$, followed by 35 cycles at $94^{\circ} \mathrm{C}$ for $1 \mathrm{~min}$, $60^{\circ} \mathrm{C}$ for $1 \mathrm{~min}, 72^{\circ} \mathrm{C}$ for $1 \mathrm{~min}$, and a final extension at $72^{\circ} \mathrm{C}$ for $5 \mathrm{~min}$. The PCR primers used were T3 5'-AATTAACCCTCACTAAAGGG-3', T7 5' - A A TACGACTCACTATAGGGA-3', and SP6 5'-AATTAGGTGACACTATA-3' (Synthetic Genetics, San Diego, CA, USA and Invitrogen, Carlsbad, CA, USA). PCR products were analyzed by electrophoresis through $1.2 \%$ agarose gels, and the images were analyzed by Kodak $^{\circledR}$ EDAS 1D software (Amersham Biosciences). DNA sequencing was conducted on a manually operated Macrophor 2010 electrophoresis system (Amersham Biosciences).

\section{Validation Test}

DNA templates from phage-recom binant control $(\mathrm{MCR}+)$ were mixed with the HeLa library before MCRPCR amplification. Phage-recombinant control templates were added in increasing amounts of $0,10,100,250$, 500, 1000, and $250 \mathrm{ng}$ while keeping the HeLa transcripts library constant at $50 \mathrm{ng}$. In the second set, the concentrations were reversed. MCR-PCR ampli- 
Table 1. Eleven cDNA Libraries with Their Respective Titer, Percent MCR, and Percent Retrieved Actin

\begin{tabular}{|cccccc|}
\hline $\begin{array}{c}\text { Library } \\
\text { No. }\end{array}$ & $\begin{array}{c}\text { Titer } \\
\text { (pfu/mL) }\end{array}$ & \% MCR & $\begin{array}{c}\text { \% Target Sequence } \\
\text { Retrieval (Actin) }\end{array}$ & $\begin{array}{c}\text { No. Blue } \\
\text { Plaques }\end{array}$ & $\begin{array}{c}\% \text { Blue } \\
\text { Plaques }\end{array}$ \\
\hline 1 & $2.1 \times 10^{9}$ & 30 & 9 & $41 / 450$ & 11 \\
2 & $1.8 \times 10^{9}$ & 13 & 22 & $22 / 350$ & 6 \\
3 & $3.0 \times 10^{9}$ & 36 & 18 & $46 / 520$ & 9 \\
4 & $2.8 \times 10^{9}$ & 12 & 20 & $22 / 220$ & 10 \\
5 & $2.4 \times 10^{9}$ & 29 & 5 & $25 / 360$ & 7 \\
6 & $3.1 \times 10^{9}$ & 8 & 22 & $95 / 630$ & 15 \\
7 & $3.6 \times 10^{9}$ & 20 & 7 & $50 / 420$ & 12 \\
8 & $2.7 \times 10^{9}$ & 6 & 12 & $25 / 280$ & 9 \\
9 & $11 \times 10^{9}$ & 8 & 26 & $40 / 340$ & 13 \\
10 & $8 \times 10^{9}$ & 7 & 25 & $10 / 190$ & 5 \\
11 & $5 \times 10^{9}$ & 20 & 8 & $34 / 310$ & 11 \\
\hline
\end{tabular}

fication was done on each of these mixtures using T7 and SP6 primers.

Plasmid-recombinant and non-recombinant controls were used in a sim ilar validation test.

\section{RESULTS AND DISCUSSION}

Figure 1 shows the PCR products from phage-recombinant and non-recombinant controls and cDNA libraries

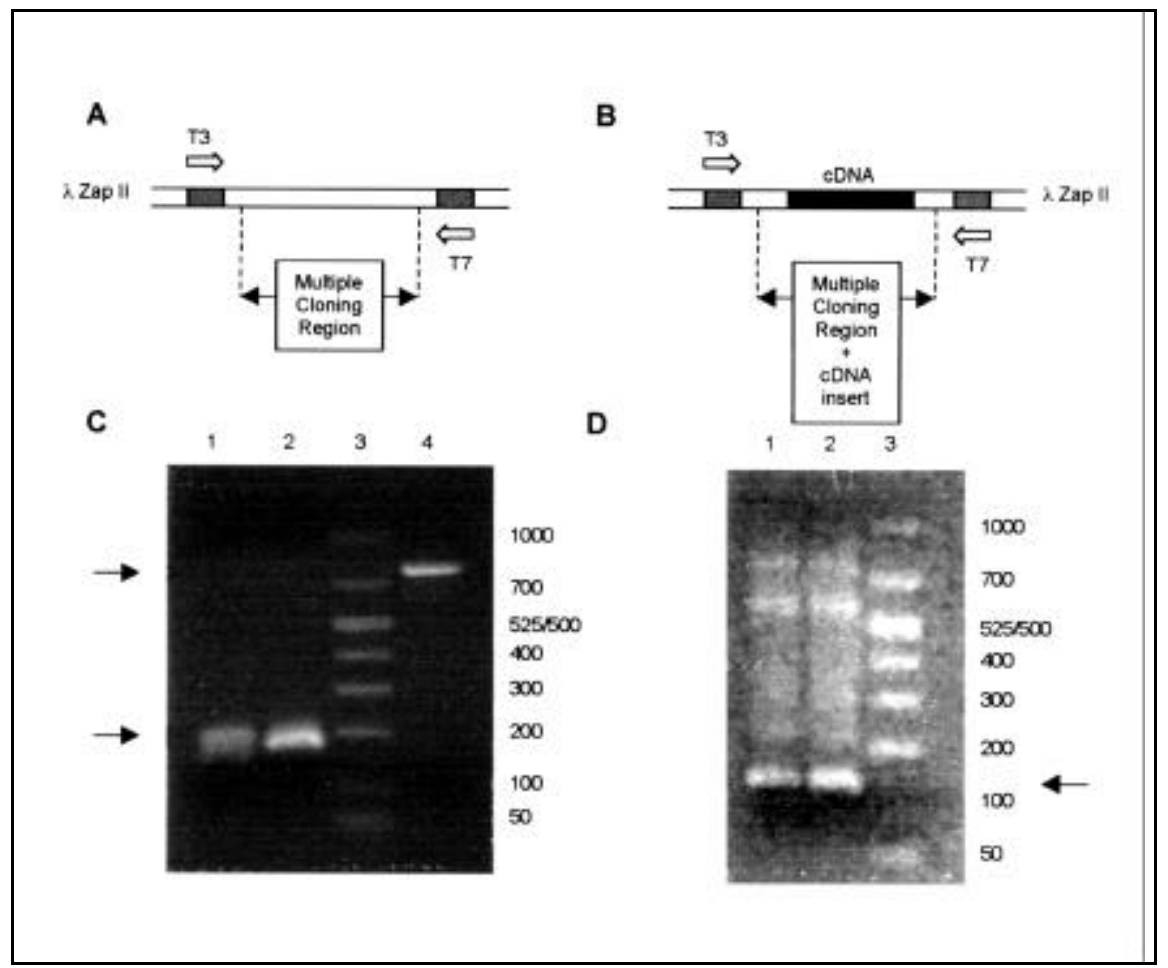

Figure 1. MCR-PCR strategy. (A) Non-recombinant parental $\lambda$ Zap II phage representing the MCR fragment. (B) Recombinant $\lambda$ Zap II phage containing a cDNA insert representing the MCR+ fragments. (C) Placental cDNA library (lane 1) and HeLa cDNA library (lane 2) showing the MCR band at $167 \mathrm{bp}$, phage recombinant control showing MCR+ band at 784 bp without any MCR band at 167 bp (lane 4). PCR molecular weight marker (Amersham Biosciences) (lane 3). (D) Placental cDNA library (lane 1) and HeLa cDNA library (lane 2) showing MCR+ fragments as smears along with the MCR band at 167 bp. PCR molecular weight marker (lane 3).

using specific transcription promoter sequences flanking the MCR as primers. Both HeLa and placental cDNA libraries show the 167-bp MCR fragment that is absent in the phage-recombinant control. On the other hand, the phage-recombinant control shows the 784-bp MCR+ fragment without the 167-bp MCR fragment (Figure 1C). The MCR+ fragments from HeLa and placental cDNA libraries are seen as smears along with the 167-bp MCR fragment following re-amplification (Figure 1D). Figure 2A shows a progressive decrease of the 167-bp MCR fragment from the HeLa cDNA library with an increase in the 784-bp MCR+ fragment of the phage-recombinant control. The result was reversed when the phage recombinant control was kept constant while varying the concentration of the HeLa library (Figure 2B). These results show that, with the increase of phage-recombinant control in the mixture, the intensity of the 167-bp MCR fragment from the HeLa library progressively decreased and vice versa. Thus, a library without parental non-recombinant phages showed only the $\mathrm{MCR}+$ fragment (Figure 2A, lane 8, and Figure 2B, lane 1). Similar results were also obtained when plasmid recombinant control was mixed with varying amounts of plasmid non-recombinant control before MCR-PCR amplification (Figure 2C). Results from these experiments validated the MCR fragment as the marker for parental non-recombinant phages in libraries.

To test the fidelity of the MCR-PCR method in detecting non-recombinant parental phages from cDNA libraries, 11 representative cDNA libraries were tested by MCR-PCR. The intensity of the MCR fragment from phage non-recombinant control was considered as $100 \%$, while compared with the intensity of the MCR fragment that was obtained from the same amount of cDNA library. Table 1 shows the titer of each of the cDNA libraries used in this study. Some of these libraries were obtained commercially, while others were obtained from different laboratories or were prepared in-house. The intensity of the 167-bp MCR-PCR fragment in these libraries differed considerably (Figure 3A). It may be noted that the libraries in Figure 3A, lanes 1, 3, 5, 7, 
and 11, show an MCR band of higher intensity, indicating the presence of a larger proportion of parental non-recombinant phages. The remaining libraries show a relatively smaller proportion of non-recombinant phages because they produce MCR bands of lesser intensities. It is noteworthy that a strict inverse relationship did not exist between the titer and the intensity of the MCR band, indicating that high titer does not always represent a nonrecombinant free library. No significant relationship was found between the titer and percent of blue/white plaques and the percent of MCR (indicating non-recombinants) in all these libraries.

To show the correlation between the MCR-PCR fragment intensity and the level of target sequence retrieval from cDNA libraries, primers of highly abundant actin transcripts were used for PCR amplification. As shown in Figure $3 \mathrm{~B}$, the intensity of the 177-bp actin fragments varied significantly. It is interesting that the libraries shown in Figure 3B, lanes 2, 4, 6, 8, 9, and 10, containing lower amounts of non-recombinant phage produced actin fragments of relatively higher intensities compared to libraries with higher MCR band intensity (Figure 3B, lanes 1, 5, and 7). However, blue/white color selection did not seem to be as conclusive as MCR-PCR as far as the percent of actin sequence retrieval was concerned. There was no direct correlation between the percent of blue plaques and the MCR band intensity (Figure 3B, lanes 3, 5, 6, and 9). The results from this study show that the presence of high proportions of parental non-recombinant phage in the library can negatively influence the level of target sequence retrieval. The presence of EcoRI linkers in multiple copies as inserts (Figure 2D) in an IPTG-X-gal-selected white plaque also demonstrates that non-recombinant phages can appear white because of false inserts or sub-optimal levels of IPTG (7). Our study shows that blue/white color selection is not always related to recombination and that libraries with high titer

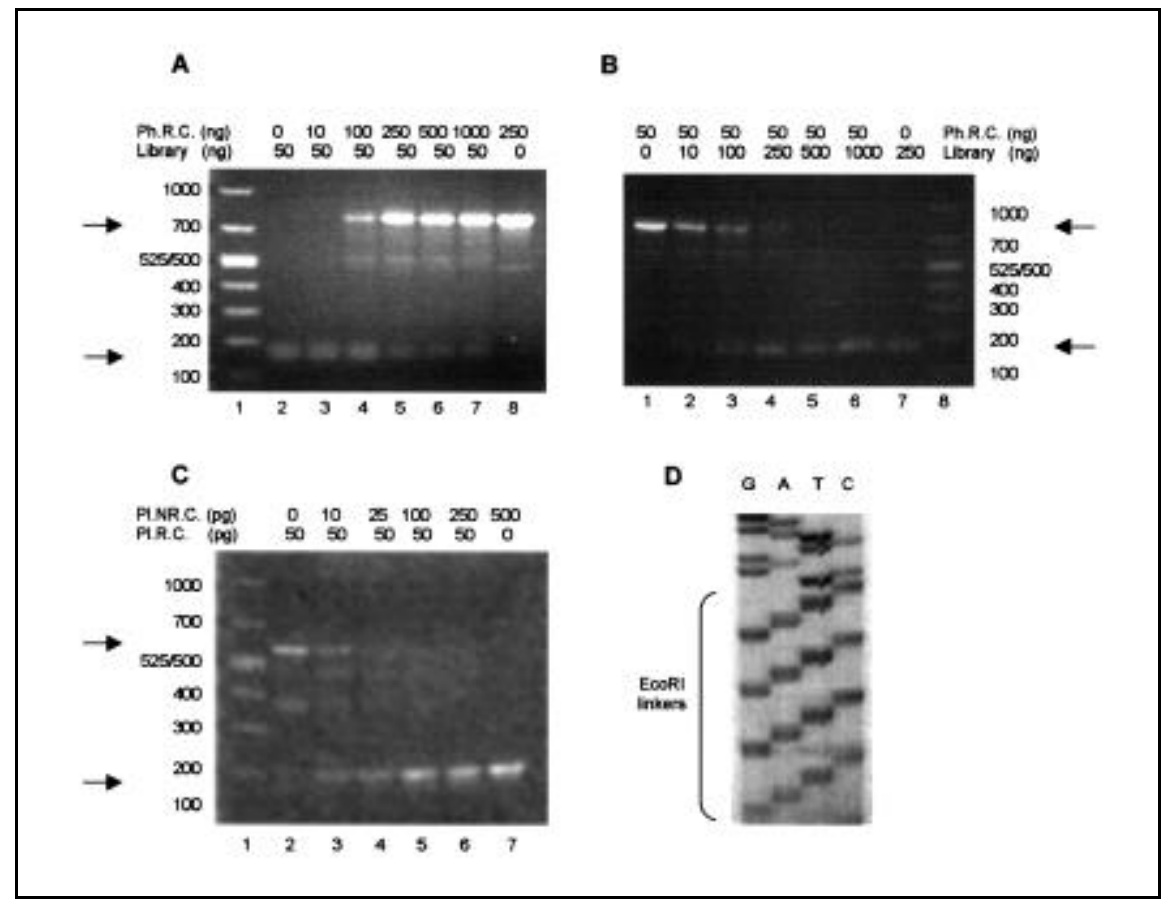

Figure 2. MCR-PCR validation. (A) MCR-PCR with increasing amounts of phage-recombinant control DNA (Ph.R.C.) mixed with HeLa cDNA library (lanes 2-8). PCR molecular weight marker (lane 1). (B) MCR-PCR with increasing amounts of HeLa cDNA library mixed with phage recombinant control (lanes 1-7). PCR molecular weight marker (lane 8). (C) MCR-PCR with increasing amounts of plasmid non-recombinant control (Pl.NR.C.) mixed with plasmid-recombinant control (PI.R.C.) (lanes 2-7). PCR molecular weight marker (Amersham Biosciences) (lane 1). (D) Partial DNA sequence of an IPTG-X-gal-selected white clone showing the presence of four EcoRI (GAATTC) linkers as a false insert in the MCR. 


\section{Short Technical Reports}

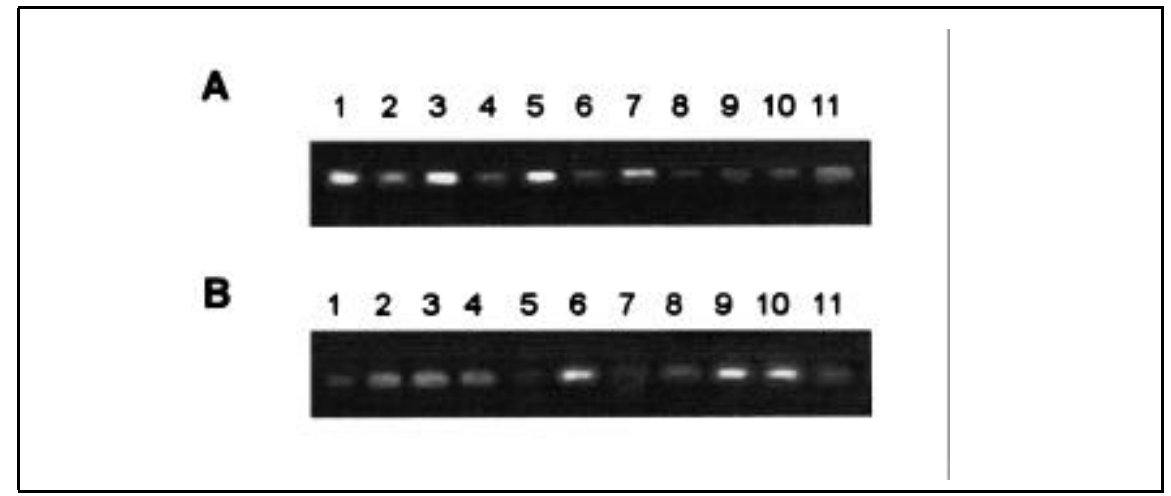

Figure 3. Detection of non-recombinants from 11 representative cDNA libraries by MCR-PCR. Equal amount of template (100 ng) was used for PCR amplification from each library. (A) Relative intensity of the MCR band from each library. (B) Relative intensity of actin band from the respective libraries.

may show a low percent of blue plaques but have both a high and low percent of target sequence retrieval (Table 1).

Taking into account the rarity (abundance) of mRNA species to be cloned, certain statistical considerations have been practiced for many years for calculating the number of unique clones that must be screened to ensure the isolation of a particular clone by using the following equation $(1,3)$.

$$
\mathrm{N}=\frac{\ln (1-\mathrm{P})}{\ln (1-\mathrm{n})}
$$

where $\mathrm{N}$ is the number of clones required, $\mathrm{P}$ is the probability of isolating the clone (usually set at a desired value of $99 \%$ ), and $n$ is the fractional proportion of the total mRNA population represented by a single mRNA species (the abundance of the desired clone).

As every cDNA library contains variable proportions of parental non-recombinant phage as background, the equation can be corrected by incorporating the MCR-PCR data as follows.

$$
\mathrm{N}=\frac{\ln (1-\mathrm{P})}{\ln (1-\mathrm{n})}(1+\mathrm{y})
$$

where, $\mathrm{N}, \mathrm{P}$, and $\mathrm{n}$ are the same as in equation 1 , and y equals the fractional proportion of non-recombinants (as represented by the MCR band intensity).

Therefore, the number of plaques to be screened as calculated by the revised formula will be proportionately higher than the number calculated by the existing method.

To our knowledge, apart from color selection, this is the first report of a PCR-based method that can identify and evaluate the presence of contaminating non-recombinants in a cDNA library. The percent of parental non-recombinants calculated by this method can be accommodated into the existing calculations for correcting the number of plaques needed for library screening. In this report, we have addressed a problem that is inherently associated with the construction and screening of a
cDNA library. The MCR-PCR method has the potential to qualify those phagecDNA ligation reactions that do not show any parental phages at the early stages of cDNA library preparation. Thus, by selecting preparations that are free of parental non-recombinant phage, one could possibly construct a cDNA library with ideal mRNA representation.

\section{REFERENCES}

1.Clarke, L. and J. Carbon. 1976. A colony bank containing synthetic Col E1 hybrid plasmids representative of the entire $E$. coli genome. Cell 9:91-99.

2.Huynh, T.V., R.A. Young, and R.W. Davis. 1988. Constructing and screening cDNA libraries in $\lambda \mathrm{gt} 10$ and $\lambda \mathrm{gt} 11$, p. 49-78. In D.M Glover (Ed.), DNA Cloning: A Practical Approach, vol I. IRL Press, Oxford.

3.Jendrisak, J., R.A. Young, and J.D. Engel. 1987. Cloning cDNA into lambda gt10 and lambda gt11. Methods Enzymol. 152:359-371.

4.Mierendorf, R.C., C. Percy, and R.A. Young. 1987. Gene isolation by screening lambda gt11 libraries with antibodies. Methods Enzymol. 152:458-469.

5.Murray, E., K. Singer, K. Cash, and R. Williams. 1993. Cloning qualified blunt-end restriction enzymes: causes and cures for light blue colonies. Promega Notes 41:1-5.

6.Sambrook, J., E.F. Fritsch, and T. Maniatis. 1989. Molecular Cloning: A Laboratory Manual. CSH Laboratory Press, Cold Spring Harbor, NY.

7.Stratagene. Zap II cDNA Synthesis Kit Instruction Manual. Stratagene, La Jolla, CA.

8.Wahl, G.M. and S.L. Berger. 1987. Screening colonies or plaques with radioactive nucleic acid probes. Methods Enzymol. 152:415-423.

9. Young, R.A. and R.W. Davis. 1983. Efficient isolation of genes using antibody probes. Proc. Natl. Acad. Sci. USA80:1194-1198.

Address correspondence to Dr. Dilip Bandyopadhyay, Division of Laboratory Medicine, Tata Memorial Hospital, Parel, Mumbai 400 012, India.e-mail: dilipsnb@vsnl.com

Received 22 December 2000; accepted 24 July 2001.

\section{Dilip Bandyopadhyay and Farhad D. Vesuna Tata Memorial Hospital Parel, Mumbai, India}

For reprints of this or any other article, contact Reprints@BioTechniques.com 